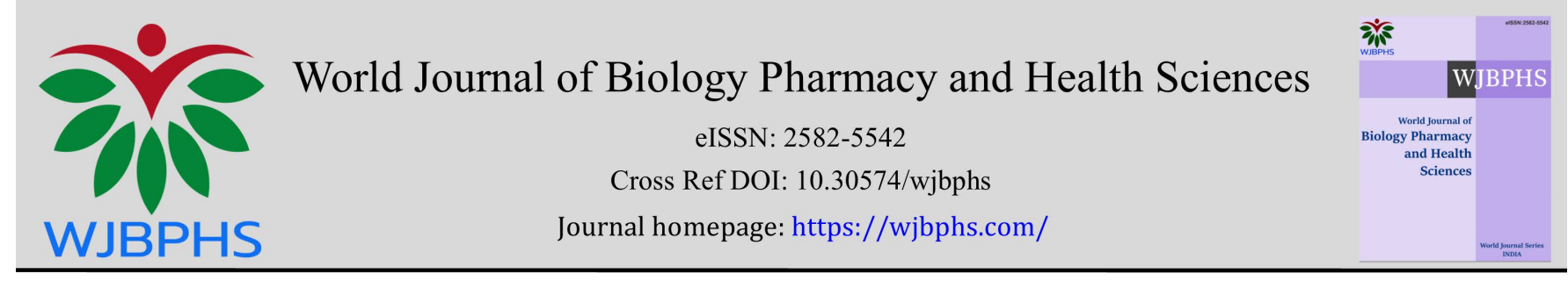

(RESEARCH ARTiCLE)

Check for updates

\title{
Knowledge and awareness about occupational therapy among healthcare professionals in Al-Ahsa
}

Senthil Vadivel *, Paramasivan Mani, Nawaf Al Anezi, Mohammed Hamad Al Subaie, Abdullah Fahad Al Mulhim, Mohammed Khalid Al Mulhim and Omar Abdullah Al Yemeni

\section{King Saud Bin Abdulaziz University for Health Sciences, College of Applied Medical Sciences, Department of Occupational therapy, Al Ahsa, Kingdom Of Saudi Arabia.}

World Journal of Biology Pharmacy and Health Sciences, 2021, 08(01), 008-012

Publication history: Received on 20 August 2021; revised on 27 September 2021; accepted on 29 September 2021

Article DOI: https://doi.org/10.30574/wjbphs.2021.8.1.0104

\begin{abstract}
Introduction and Aim: Occupational Therapy (OT) is a health care profession in which assist persons with physical, mental, or cognitive impairments through the therapeutic use of daily activities. In addition, the therapeutic use of everyday life activities (occupations) is aimed to enable the individual or groups to involve them in roles, customs and routines in home, college, place of business, society, and other settings by occupational therapist [1]. OT considers the only health care profession that enables people to do the activities they want and important for them via the therapeutic use of everyday activities [2]. Occupational therapists capacitate people of all ages to assist them by enhancing their health and inhibiting or adjusting with the injury, illness, or disability [3]. The aim of the study is to evaluate the knowledge of Occupational Therapy among healthcare professionals of National Guard Health Affairs in Al-Ahsa.
\end{abstract}

Method: The study is cross-sectional study; the research was conducted in National Guard Health Affairs in Al-Ahsa. The healthcare provider's knowledge about OT was assessed by the survey. The questionnaire consisted of seventy-five closed statements in five different aspects. The five-point Likert Scale (1=strongly disagree and 5=strongly agree) was used to measure the healthcare provider's knowledge about OT.

Results: The total numbers of participants were 218 participants, and 167 participants completed the survey showing a high response rate of $76.6 \%$. The study found that the total knowledge among healthcare professionals about OT is moderate.

Conclusion: The healthcare professionals of National Guard Health Affairs in Al-Ahsa have a moderate to minimal knowledge about OT. Further studies are needed on the importance of developing OT in the Kingdom of Saudi Arabia in general and in Al-Ahsa in particular.

Keywords: Occupational Therapy; Awareness; Knowledge; Healthcare

\section{Introduction}

Occupational therapy (OT) considers one of the most important elements of a multidisciplinary team approach that emphasizes on enabling involvement in significant occupation as a crucial component of healthcare. OT has an important role in interdisciplinary and multidisciplinary healthcare service team. Mainly, the awareness of OT has been indistinct among the public and healthcare professionals. Awareness about OT among healthcare professionals is important to guarantee the conveyance of inclusive, holistic, and standard care services [4]. Awareness and knowledge

\footnotetext{
${ }^{*}$ Corresponding author: Senthil Vadivel

King Saud Bin Abdulaziz University for Health Sciences, College of Applied Medical Sciences, Department of Occupational therapy, Al Ahsa, Kingdom Of Saudi Arabia.
} 
about OT is essential between other healthcare givers to ensure that the quality of care service is provided comprehensively and holistically. Healthcare givers should know and understand other team member's roles to prevent role confusion and the referrals can be made correctly and in appropriate way. Therefore, it is important for healthcare givers to have the knowledge about OT. Healthcare professionals must comprehend other team member's roles in which proper referrals are made and to prohibit role confusion [5]. Sometimes, clients can be unaware of OT services because of the lack of knowledge and awareness that other healthcare givers have about the services that the occupational therapy practitioners (OTP) provide. Public awareness about OT influences the procedure of clinical reasoning among OTPs. Supervisors have inaccurate expectations about the role of OTPs in return, influences the procedure of treatment planning and reduce the standard outcomes of OT intervention, The lack of awareness in OT profession from healthcare professionals decreases the chances of involving in reciprocal planning and reasoning with OTPs which indicates negatively on the standard of care. The level of awareness of healthcare professionals about Occupational therapy services will improve the mutual planning and better outcomes of the client's intervention [6]. The 0TPs assist individuals of all ages from new-born to older adult. Furthermore, OTPs work with individuals who suffer from physical, cognitive, and emotional disabilities which hinder their capabilities to do meaningful activities like eating, dressing, driving, writing, etc. It is important for other professions to be aware and have the knowledge of OT services.

The need of OT in hospitals and healthcare facilities is important. As human beings, we do activities and occupations that are meaningful for us. Occupational therapist has the knowledge to analyze and create the patient's activities after the injury. Amy Lorino said that "Occupational therapy services positively impact interdisciplinary care and enhance patient participation, satisfaction, and clinical outcomes" [7]. Also, according to the best practice \& research Clinical rheumatology "Occupational Therapy, designed to improve participation in activities and roles, should theoretically impact on health status in the longer term, improve quality of life and reduce health care and social costs" [8]. The Occupational therapist has the ability to treat and help all age groups to reach the independency. Therefore, the existence of OT in the healthcare facilities is essential because investing in OT has the potential to develop care quality and reduce readmission rates [9].

There are many studies that have conducted worldwide about OT awareness such as, Makkah, Jordan and Nigeria. The study that was conducted in Makkah was planned to evaluate the Health Care Professionals information about OT. The result of the study says that Healthcare professionals in Makkah have shortage of information about OT of its purpose and treatment techniques. This dearth of information needs to be addressed to develop the provision of service for patients in need of OT [10]. The study that was held in Jordan was designed to assess the Jordanians awareness about OT. The study concludes a lack of understanding of the OT role between common people, other healthcare professionals, and even the patient's themselves. Also, OT programs should be inserted within the curriculums and situations of all Healthcare professionals [11]. Moreover, the study that had done in Nigeria was to assess the Nigerian Medical and Health Science Undergraduates awareness and knowledge about OT. The result shows that Nigerian Medical and Health Sciences Undergraduates own high awareness but have little to average information about the OT profession, job position, and work status [12]. OT needs the awareness by any member of the healthcare profession. Furthermore, the study in the British Journal of OT found that "It is probable that there will always be a few who will deny all knowledge of the occupational therapist, despite much assistance from and exposure to the profession" [13]. The study in the Journal of Allied Health about health science students' awareness of OT, conclude that there is a lack in specifics of knowledge about the OT profession [14].

There are no research that conducted or measured the knowledge of healthcare professionals about OT in Al-Ahsa. The level of awareness about OT among health professionals is important in clinical setting. Therefore, our aim is to evaluate the knowledge of Occupational Therapy among healthcare professionals of National Guard Health Affairs in Al-Ahsa.

\section{Methodology}

The study is cross-sectional study because the data were collected at given point across the study. Since the data are numerical, the research is quantitative research. The study was conducted in National Guard Health Affairs in Al-Ahsa. We conducted the study in Six months. The Healthcare provider's knowledge about OT was assessed by the survey. The questionnaire was addressed as outline survey. The purpose of the questionnaire was to measure the healthcare professionals' knowledge about OT in National Guard Health Affairs in Al-Ahsa. The permission was taken from the hospital ethical committee. We have contacted with healthcare providers in King Abdulaziz Hospital in Al-Ahsa to send the link through their emails. We used a developed structured questionnaire from research have been conducted in Makkah called "Knowledge About Occupational Therapy in Makkah, Saudi Arabia. Where Do Health Care Professionals Stand?". The questionnaire consisted of fifty-four closed statements in different aspects, including knowledge about goals of OT practice, knowledge about OT treatment methods and media. The five-point Likert Scale (1=strongly disagree and $5=$ strongly agree) was used to measure the healthcare professionals' knowledge about OT. The study 
populations were the healthcare providers in King Abdul-Aziz Hospital in Al-Ahsa. Moreover, by using the Roasoft calculator, we calculated the estimated sample size. The response distribution was estimated to be $50 \%$ on the healthcare providers' knowledge about OT with confidence level at 95\% and margin of error of 5\%. We asked the Human Resource in King Abdulaziz Hospital in Al-Ahsa to give us an estimated number of health care providers which is 500. After we have done the calculation, we found that the estimated sample size based on our estimated population is 218. All the adult healthcare providers either male or female who meet the following criteria were included. The inclusion criteria were Physicians, Nurses and Allied health professionals. In other hand, the exclusion criteria administrative staff and medical rehabilitation department staff. The medical rehabilitation department staff was excluded because they have direct contact with OTPs.

\section{Results}

All the participants who met the inclusion criteria in King Abdul-Aziz Hospital in Al-Ahsa was recruited in the study. The total numbers of participants were 218 participants, and 167 participants completed the survey showing a high response rate of $76.6 \%$. As it shown in (table 1), $2.1 \%(n=2)$ of the participants their level of education is Diploma which is considered the lowest percentage between the participants. While the highest percentage of the participants their level of education is Bachelors (86.1\%, $n=144)$. 7.3\% $(n=13)$ of the participants were having PhD's degree and 4.5\% $(n=8)$ were having master's degree. Most of the participants were Physicians $47.3 \%(n=79)$, whereas $21.4 \%(n=35)$ were Nurses. 31.3\% ( $n=52)$ of the participants were other Allied health professions. $71.2 \%(n=119)$ of the participants have not used OT services, while There were $28.8 \%(n=48)$ clients who had received OT services.

The participants answered section two completely, which was about the knowledge about goals of OT. Most of the participants agreed that occupational therapist helps the clients on walking and using hand. 66.7\% ( $\mathrm{n}=112)$ of the participants agreed that occupational therapist helps on developing cognitive skills, while 5.4\% ( $n=9$ ) of the participants disagreed. $62 \%(n=104)$ of the participants agreed that the occupational therapy can improve the social interaction of the service recipient. $48.2 \%(n=81)$ of the participants were agreed that the OT can help the service recipient to find appropriate job. On contrast, $22 \%(n=37)$ disagreed that occupational therapist plays important role in finding appropriate job for the client. Most of the participants agreed that occupational therapist works within medical and academic team. However, $14.3 \%(n=24)$ had disagreed that occupational therapist works within academic team indicating slight lack of knowledge about OT goals in the academic filed. $39 \%(n=66)$ were neutral about the occupational therapist role includes integrating children with special needs in the mainstream schools.

The result on section two was mainly about knowledge about OT treatment methods and media. 70.8\% (n=117) of the participants agreed that the occupational therapist implements needed modifications on service recipients' environment, while $4.8 \%(n=8)$ of them disagreed. Quarter of the participants $30.4 \%(n=51)$ had disagreed on occupational therapist to provide psychological consultations and 34.5\% $(n=58)$ of them were neutral. Moreover, almost half of the participants agreed about the occupational therapist work with service recipient to improve the function of the upper extremities and the physical therapist works to improve the function of the lower extremities. Also, around $70.2 \%(n=118)$ agreed that the occupational therapist prescribes and modifies assistive devices that the service recipients use to perform at an optimal level of functioning, while $23.8 \%(n=33)$ were neutral.

Table 1 The characteristics of the participants

\begin{tabular}{|l|c|}
\hline \multicolumn{1}{|c|}{ Level of education } & Numbers (parentage) \\
\hline Diploma & $2(2.1 \%)$ \\
\hline Bachelors & $144(86.1 \%)$ \\
\hline Master & $8(4.5 \%)$ \\
\hline PhD & $13(7.3 \%)$ \\
\hline Profession & \\
\hline Physician & $79(47.3 \%)$ \\
\hline Nurse & $36(21.4 \%)$ \\
\hline Allied health profession & $52(31.3 \%)$ \\
\hline
\end{tabular}


As shown in Figure 1, 12\% of the sample had poor or no knowledge about OT. Also, we found that there was no significant difference in knowledge about OT amongst the three professions $(\mathrm{P}=0.125>0.05)$. Also, we found that there was no significant difference in knowledge about OT among the level of education $(\mathrm{P}=0.290>0.05)$.

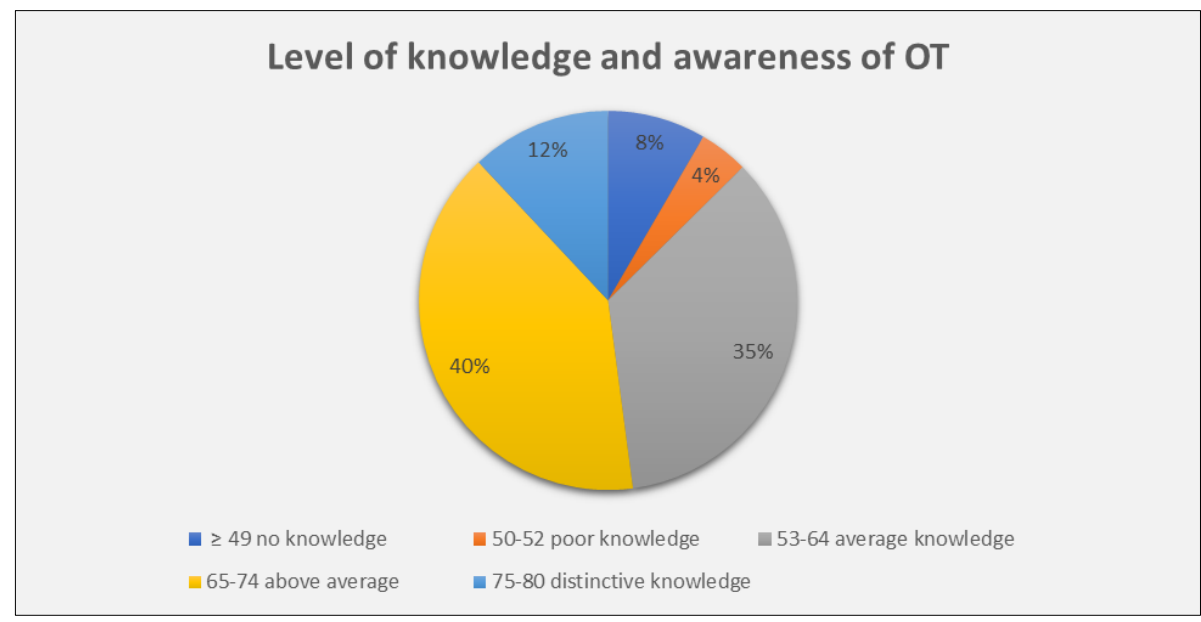

Figure 1 The Circle diagram shows the percentage of the level of knowledge and awareness of OT

\section{Discussion}

This research was conducted in order to discover the Knowledge and awareness about Occupational Therapy among healthcare professionals in Al-Ahsa. The questionnaire has been done to find the knowledge and awareness that the healthcare professionals have about OT. The participants of the questionnaire were included physicians, nurses, and allied health professions. The questionnaire was given in both Arabic and English languages to include and to be fair with all the Arabic and the English speakers. The study found that the total knowledge among healthcare professionals about OT is moderate. According to the conducted descriptive statistics, OT is still not well recognized in Al-Ahsa and especially in King Abdul-Aziz Hospital. Therefore, the result was in between (in the middle). This was a predicted result because OT is new major in eastern province of Saudi Arabia generally and in Al-Ahsa in particular. Also, the lack of universities that have OT program is one of the major effects of this study. However, it is not well known enough to know about the goals and implications of OT. Although the result was moderate, we still need more awareness that promote the knowledge of OT in the area. The awareness is needed to be spread among healthcare professionals and the public and to make sure that everyone knows what OT can provide.

Our study found that $48.2 \%$ of the participants were agreed that the OT can help the service recipient to find appropriate job. This is consistent with the finding of other study done in Makah, which found that above 59\% of health care providers agreed that the occupational therapist can help other persons to find appropriate job. It is comprehensible, as the word "occupation" may have been related to work, which is also its literal sense. 66.7\% of the participants agreed that occupational therapist helps on developing cognitive skills. This is different with the finding of other study done in Jordan, which found that there was a limited understanding of the function of OTPs in mental and psychiatric health environment. Around $70.2 \%$ agreed that the occupational therapist prescribes and modifies assistive devices that the service recipients use to perform at an optimal level of functioning. Assistive device is any equipment that used to increase, maintain, or improve the functional capabilities of individuals with disabilities. Therefore, assistive device is also a part of the provision of OT services in the area of practice. $39 \%$ were neutral about the occupational therapist role includes integrating children with special needs in the mainstream schools. It is consistent with the finding of other study done in Jordan, which found that the importance of OT in school-based settings was not fully understood. Most of the participants agreed that occupational therapists work within medical and academic team and it mirrors the result that found in Jordan, which attributed that there was lack awareness due to the deficiency of shared education and discussion chances among members of the multidisciplinary team. In conjunction with other members of the health team, OTPs has a responsibility and an obligation to set targets and action plans.

\section{Limitations}

The results of this study support the research assumptions and answers to the study questions. Although the results of this study were conducted, there are many limitations that it faced. First, the sample participants were limited, as the research was limited to Physicians, Nurses, and Allied health professions at King Abdulaziz Hospital in Al-Ahsa. Second, 
the participants in the study were only 218 because of the limited time. Moreover, some of the participants declined to participate in the study, which affected the limitations of this study. Third, the participants are not devoted to the study, and their busy work and many tasks have an impact on the participation rate.

Finally, due to the limitations of this study, future studies are suggested to help increase awareness of occupational therapy. Also, students and researchers can do further future studies on the importance of developing occupational therapy in the Kingdom of Saudi Arabia in general and in Al-Ahsa in particular. Moreover, we suggest educating high school graduates and their parents about the importance of joining the Occupational Therapy Department and encouraging them to do so. All these help in developing the university and society and aims to provide better health care for all.

\section{Conclusion}

The healthcare professionals of National Guard Health Affairs in Al-Ahsa have a moderate to minimal knowledge about OT. Further studies are needed to enhance the awareness about OT importance in health care team and common public in the Kingdom of Saudi Arabia in general and in Al-Ahsa in particular.

\section{Compliance with ethical standards}

\section{Acknowledgments}

We would like to acknowledge with much appreciation to our university Research committee, King Abdullah International Medical Research Center and our Occupational therapy Students (Mohammed Hamad Al Subaie, Abdullah Fahad Al Mulhim, Mohammed Khalid Al Mulhim and Omar Abdullah Al Yemeni). Also, we would like to thank Dr. Abdullah Al Ghamdi for his help and support to find the approximate sample size. We would like to show our deepest appreciation to Dr. Mohammed Al Mohaini, Research Methodology course coordinator for his continuous advice and contribution to our project.

\section{Disclosure of conflict of interest}

We have no conflict of interests between all the authors for publication.

\section{Statement of informed consent}

The informed consent form prior approved by King Abdullah International Medical Research Center and Informed consent was obtained from all individual participants included in the study.

\section{References}

[1] American Occupational Therapy Association. Occupational Therapy Practice Framework: Domain Et Process.

[2] Internet]. 2021 [cited 1 October 2021]. Available https://www.caot.ca/site/aboutot/whatisot?nav=sidebar

[3] [Internet]. Aota.org. 2021 [cited 1 October 2021]. Available from: https://www.aota.org/About-OccupationalTherapy.aspx

[4] Code of Ethics and Professional Conduct for Occupational Therapists: College of Occupational Therapists, 2001 [Internet]. SAGE Journals. 2021 [cited 1 October 2021]. Available from: https://journals.sagepub.com/doi/abs/10.1177/030802260106401207?journalCode=bjod

[5] Dunford C, Street E, O'Connell H, Kelly J, Sibert JR. Are referrals to occupational therapy for developmental coordination disorder appropriate?. Archives of disease in childhood. 2004 Feb 1;89(2):143-7.

[6] Shafaroodi N, Kamali M, Parvizy S, Mehraban AH, O’Toole G. Factors affecting clinical reasoning of occupational therapists: a qualitative study. Medical journal of the Islamic Republic of Iran. 2014;28:8.

[7] Lorino A. When Do Patients Need Occupational Therapy?. Home healthcare now. 2018 Nov 1;36(6):395.

[8] Hammond A. What is the role of the occupational therapist?. Best practice \& research Clinical rheumatology. 2004 Aug 1;18(4):491-505. 
[9] Rogers AT, Bai G, Lavin RA, Anderson GF. Higher hospital spending on occupational therapy is associated with lower readmission rates. Medical Care Research and Review. 2017 Dec;74(6):668-86.

[10] Meny AH, Hayat AA. Knowledge about occupational therapy in Makkah, Saudi Arabia. Where do health care professionals stand. Int Ann Med. 2017;1(11):6.

[11] Darawsheh WB. Awareness and knowledge about occupational therapy in Jordan. Occupational therapy international. 2018 May 21;2018.

[12] Olaoye OA, Emechete AA, Onigbinde AT, Mbada CE. Awareness and knowledge of occupational therapy among Nigerian medical and health sciences undergraduates. Hong Kong Journal of Occupational Therapy. 2016 Jun;27(1):1-6.

[13] McAvoy E. Occupational who? Never heard of them! An audit of patient awareness of occupational therapists. British Journal of Occupational Therapy. 1992 Jun;55(6):229-32.

[14] Alotaibi N, Shayea A, Nadar M, Tariah HA. Investigation into health science students' awareness of occupational therapy: Implications for interprofessional education. Journal of allied health. 2015 Mar 2;44(1):3-9. 